\title{
Menu labeling implementation in dine-in restaurants: the Public's knowledge, attitude and practices
}

\author{
Hadia Radwan ${ }^{1 *}$ (D) Eman M. Faroukh² and Reyad Shaker Obaid ${ }^{1}$
}

\begin{abstract}
Background: The practice of menu labeling is gaining popularity worldwide as a potential policy to reduce energy intake as a means to decrease the prevalence of obesity. So the purpose of the present study is to identify the knowledge, attitudes, and practices of adults regarding the implementation of menu labeling in dine-in restaurants.

Methods: A cross sectional survey included 2020 male or female adults (aged $\geq 18$ years old) participants was collected from two cities in the United Arab Emirates(UAE). The participants filled a validated questionnaire in public places in two cities. A chi-squared test was conducted to compare responses for differences in proportions.

Results: Most participants were knowledgeable about energy requirements for moderately active men (60\%) and women (59\%), but underestimated energy requirements for inactive adults (34\%). The majority of the respondents favored the requirement to post calorie information on menus of dine-in restaurants at the point of purchase (76\%). About half the respondents (48\%) were more likely to visit restaurants with labeled menus.

Conclusion: The results from this study may form the basis for future strategies in mandating calorie labeling of restaurant menu items in UAE. Menu labeling may be a useful policy tool for promoting appropriate caloric consumption.
\end{abstract}

Keywords: Menu labeling, Nutrition, Restaurants, Knowledge, Attitude, Practice

\section{Background}

Excessive eating and unhealthy food selections are causative factors in one of the most persistent health apprehensions facing the Gulf countries. The latest research investigating the prevalence of obesity reveals that approximately one quarter of adults in the Gulf countries, $28.52 \%$ of women and $15.5 \%$ of men over the age of 20, are obese [1]. According to a recent report from the Health Authority in Abu Dhabi, obesity may account for up to $60 \%$ of nationals in the United Arab Emirates (UAE) [2]. An area of emerging importance as a policy with the potential to reduce the widespread occurrence of obesity is the display of calorie information on restaurant menus in both dine-in and fast food chain restaurants [3].

\footnotetext{
* Correspondence: hradwan@sharjah.ac.ae

'Department of Clinical Nutrition and Dietetics, College of Health Sciences/ Sharjah Institute for Medical Research, University of Sharjah, P. O Box 27272, Sharjah, United Arab Emirates

Full list of author information is available at the end of the article
}

Menu labeling is one strategy among a broad spectrum of efforts to reduce rates of obesity and its comorbidities. Studies have shown that $90 \%$ of individuals ordering from restaurants underestimate the amount of energy within meals by as much as $600 \mathrm{kcal}$ [4]. In the United States, a federal law obligates restaurant chains with 20 or more outlets to post nutrition information on their menus $[5,6]$. Many studies have shown that the public is interested in knowing the number of calories present in the meals they order from restaurants [7]. Moreover, Spicer [8] showed that promoting the use of calorie information, and calorie awareness, supports lower-calorie choices at fast food eateries. Caloric labeling is proposed as an innovative approach that will change the food environment, and increases customers' awareness of calories, which may, in turn, help to lower the costs of the obesity epidemic [9].

Energy-dense foods consumed in restaurants are generally higher in saturated fat, cholesterol and sugar. A 
transformation in the pattern of consumption has led to the creation of an obesogenic environment, and an increase in the prevalence of lifestyle diseases such as cardiovascular diseases and diabetes [10]. The share of daily calories consumed in restaurants and fast food establishments was reported to have increased from 6 to $20 \%$ between 1977 and 78 and 2005-08 [11].

Consumers' knowledge of, and their ability to estimate, calorie count, and the fat, saturated fat, and sodium content of food was investigated by Burton et al., [10]. The actual fat and saturated fat levels were found to be higher than consumers' estimates [12], and menus lack the information that gives consumers the capacity to choose more healthful foods.

The purpose of the present study is to identify the knowledge, attitudes, and practices of adults regarding the implementation of menu labeling in dine-in restaurants.

\section{Methods}

The study was conducted in the cities of Sharjah and Dubai in the United Arab Emirates and approved by Ethical Committee at the University of Sharjah. Consent was obtained from participants before they were interviewed.

\section{Target population}

A convenient sample of 2020 participants was collected from the two cities. The criterion for inclusion was being an adult male or female aged $\geq 18$ years old. Data was collected using a questionnaire in different clusters in heavily populated areas. The questionnaires were distributed in public places such as parks, malls, educational institutes, neighborhoods, governmental buildings, and coffee shops in Sharjah and Dubai.

It is worth noting that UAE has a high entry rate record from secondary to higher education [13]. It was reported that about $95 \%$ of all girls and $80 \%$ of boys finished their secondary high school year to apply for admission to a higher education institution.

\section{Study design}

The study was a quantitative, cross-sectional study that used a relevant validated questionnaire [4]. Some modifications to the questionnaire were made in order to identify the percentage of participants having nutritional awareness and who were interested in the implementation of calorie information on restaurant menus.

\section{Data collection}

A pilot study using the questionnaire was conducted to assess if any changes were required. The main questionnaire was subsequently adjusted by adding a third option, "Neither", to the question asking whether the participants favor or oppose the government mandating menu labeling
The questionnaire was tested for validity by a panel composed of four professionals in the Clinical Nutrition and Dietetics Department. The questionnaire was divided into four main sections: background information, knowledge, attitude and practices. The background information included age, gender, nationality, city of residence, and the frequency of dining outside the home. Participants' knowledge was tested by asking about the estimated daily energy requirements for each gender and for their different physical activity levels (active and inactive). Attitude of the population sample measured the extent to which menu labeling was important to the participant, whether they supported menus being labeled, and if so, whether stamping or labeling menus with caloric values was preferred. Practices assumed that dine-in restaurant menus were labeled and participants were asked if they would choose labeled menu restaurants, select foods that are lower in calories, and whether their selection of foods was based on cost, appetite or caloric content.

The questionnaire was distributed along with the consent form and their contents were explained to the participants. The consent form, signed by the participants before completing the questionnaire, provided the option to accept or refuse participation in the study.

\section{Statistical analysis}

Data was analyzed by using Statistical Package for the social sciences (SPSS) version 21.0(Statistical Package for Social Sciences, Version 21). Descriptive analysis, frequencies and percentages were calculated for demographic data. Chi-square test was used to study the correlation between two variables. Differences were considered significant at $p<0.05$.

\section{Results}

Characteristics of the study sample

Approximately half of the respondents were females, under 24 years of age (60\%), non-Emirati Arab (62\%), living in Sharjah (52\%), and had education more than a high school degree (76\%). About one third (36\%) of respondents reported visiting dine-in restaurants one to three times per month (Table 1).

\section{Knowledge of daily caloric requirements}

Most of the respondents were able to identify the correct caloric intake for moderately active men $(60 \%)$ and for moderately active women (59\%) which, according to federal dietary guidelines, is between 1500 and less than $3000 \mathrm{kcal}$ [14]. However, only $34 \%$ of participants were aware of the caloric requirements for inactive adults (Table 2).

The participants' estimation of the correct daily energy requirements for moderately active men - between 
Table 1 Frequency distribution of the general characteristics of the participants $(N=2020)$

\begin{tabular}{|c|c|c|}
\hline Variables & Percent & Number \\
\hline \multicolumn{3}{|l|}{ Gender } \\
\hline Male & 43 & 870 \\
\hline Female & 57 & 1146 \\
\hline \multicolumn{3}{|l|}{ Nationality } \\
\hline Emirati & 21 & 422 \\
\hline Non Emirati Arab & 62 & 1237 \\
\hline Non Arab & 17 & 333 \\
\hline \multicolumn{3}{|l|}{ Age } \\
\hline $18-24$ & 60 & 1193 \\
\hline $25-39$ & 28 & 571 \\
\hline $40+$ & 12 & 241 \\
\hline \multicolumn{3}{|l|}{ Education Level } \\
\hline High School or less & 24 & 477 \\
\hline More than High school & 76 & 1525 \\
\hline \multicolumn{3}{|l|}{ City of Residence } \\
\hline Abu Dhabi & 13 & 263 \\
\hline Dubai & 24 & 485 \\
\hline Sharjah & 52 & 1035 \\
\hline Other Cities & 11 & 216 \\
\hline \multicolumn{3}{|l|}{ Restaurant Visit } \\
\hline Never & 3 & 68 \\
\hline 1-3 times per month & 36 & 715 \\
\hline 1-2 times per week & 35 & 700 \\
\hline 3-4 times per week & 16 & 329 \\
\hline Once Daily & 10 & 195 \\
\hline
\end{tabular}

Table 2 Percentage distribution of the participants who knew the caloric recommendations of the different levels of activities for the men and women ( $N=2020)$

\begin{tabular}{lll}
\hline Variables & Percent & Number \\
\hline $\begin{array}{l}\text { Moderately Active Men } \\
\text { Caloric Recommendations } \\
\text { Answered Correctly }\end{array}$ & 60 & \\
$\quad$ Answered Incorrectly & 40 & 1216 \\
Moderately Active Women & & 798 \\
Caloric Recommendations & 59 & 1186 \\
Answered Correctly & 41 & 824 \\
Answered Incorrectly & & \\
Inactive Adults Caloric & & \\
Recommendations & 34 & 1319 \\
$\quad$ Answered Correctly & 66 & \\
Answered Incorrectly & &
\end{tabular}

$1500-3000 \mathrm{Kcal}$ - differed significantly by gender, nationality, and education $(P<0.05)$. Similarly, estimations of the correct daily energy requirements for moderately active women $(1500-3000 \mathrm{Kcal})$ were significantly different by age $(P<0.01)$. Moreover, the correct estimation of energy requirements for inactive adults differed significantly by gender, nationality, and city of residence $(P<0.05)$ (Table 3$)$.

\section{Attitudes towards menu labeling}

The majority of the participants reported that menu labeling was very useful (47\%) and somewhat useful (35\%) (Table 4) and favored mandatory menu labeling (76\%) (Table 5).

Age, gender and nationality are shown to be significant variables associated with the participants' attitudes towards menu labeling. In particular, young females of non-Emirati Arab nationality are seen to favor menu labeling in dine-in restaurants $(P<0.005)$ (Table 6).

\section{Future practices towards menu labeling}

When participants' were asked about the likelihood of dining at a menu-labeled restaurant, $48 \%$ replied that they will more likely, to choose to dine in a restaurant that has menu labeled (Table 7).

The association between demographic variables of the participants and their likeliness to eat at a menu-labeled dine-in restaurant is shown. Significant differences by age, city of residence and education $(P<0.005)$ were reported for these practices (Table 8).

\section{Discussion}

This study has found that most participants estimated correctly the energy requirements for moderately active men and women, but tended to underestimate energy requirements for inactive adults. They expressed a positive attitude towards menu labeling in dine in restaurants; a majority viewed it as very or somewhat useful, reported being more likely to eat in a restaurant with menu labeled restaurant.

The findings of this study were consistent with those of Bleich \& Pollack [4], who found that the American adult population was well informed about energy requirements for moderately active men and women, but tended to underestimate energy requirements for inactive adults. That study also reported that Americans expressed a positive attitude towards calorie posting in chain restaurants; a majority viewed it as very useful or somewhat useful. In the current study, the majority of the participants favored menu labeling, stating that having menus provide caloric labeling in dine-in restaurants is very useful.

The results of this study also showed a positive correlation between age and likeliness to eat at a menu- 
Table 3 Percentage of the participants who expressed knowledge of energy requirements of active and inactive men or women $(N=2020)$

\begin{tabular}{|c|c|c|c|c|c|c|c|c|c|c|c|c|}
\hline \multicolumn{13}{|c|}{ Knowledge of energy requirements } \\
\hline \multirow[t]{2}{*}{ Variable } & \multicolumn{4}{|c|}{ Moderately active men } & \multicolumn{4}{|c|}{ Moderately active women } & \multicolumn{4}{|c|}{ Inactive adults } \\
\hline & $<1500$ & $\begin{array}{l}1500- \\
3000\end{array}$ & $\begin{array}{l}3000- \\
4500\end{array}$ & $>4500$ & $<1500$ & $1500-3000$ & $\begin{array}{l}3000- \\
4500\end{array}$ & $>4500$ & $<1500$ & $1500-3000$ & $\begin{array}{l}3000- \\
4500\end{array}$ & $>4500$ \\
\hline \multicolumn{13}{|l|}{ Age, $Y$} \\
\hline $18-24$ & $(8.8 \%)$ & $\begin{array}{l}720 \\
(60.4 \%)\end{array}$ & $\begin{array}{l}343 \\
(28.8 \%)\end{array}$ & $\begin{array}{l}25 \\
(2.1 \%)\end{array}$ & $\begin{array}{l}340 \\
(28.5 \%)\end{array}$ & $\begin{array}{l}725 \\
(60.8 \%)\end{array}$ & $\begin{array}{l}113 \\
(9.5 \%)\end{array}$ & $\begin{array}{l}14 \\
(1.2 \%)\end{array}$ & $\begin{array}{l}638 \\
(53.5 \%)\end{array}$ & $\begin{array}{l}407 \\
(34.1 \%)\end{array}$ & $\begin{array}{l}101 \\
(8.5 \%)\end{array}$ & $\begin{array}{l}46 \\
(3.9 \%)\end{array}$ \\
\hline $25-39$ & $\begin{array}{l}62 \\
(10.9 \%)\end{array}$ & $\begin{array}{l}337 \\
(59.1 \%)\end{array}$ & $\begin{array}{l}156 \\
(27.4 \%)\end{array}$ & $\begin{array}{l}15 \\
(2.6 \%)\end{array}$ & $\begin{array}{l}191 \\
(33.6 \%)\end{array}$ & $\begin{array}{l}301 \\
(52.9 \%)\end{array}$ & $\begin{array}{l}66 \\
(11.6 \%)\end{array}$ & $\begin{array}{l}11 \\
(1.9 \%)\end{array}$ & $\begin{array}{l}310 \\
(54.9 \%)\end{array}$ & $\begin{array}{l}194 \\
(34.3 \%)\end{array}$ & $\begin{array}{l}43 \\
(7.6 \%)\end{array}$ & $\begin{array}{l}18 \\
(3.2 \%)\end{array}$ \\
\hline $40+$ & $\begin{array}{l}23 \\
(9.7 \%)\end{array}$ & $\begin{array}{l}153 \\
(64.6 \%)\end{array}$ & $\begin{array}{l}56 \\
(23.6 \%)\end{array}$ & $\begin{array}{l}5 \\
(2.1 \%)\end{array}$ & $\begin{array}{l}65 \\
(27.7 \%)\end{array}$ & $\begin{array}{l}151 \\
(64.3 \%)\end{array}$ & $\begin{array}{l}15 \\
(6.4 \%)\end{array}$ & $\begin{array}{l}4 \\
(1.7 \%)\end{array}$ & $\begin{array}{l}135 \\
(56.7 \%)\end{array}$ & 85 (35.7\%) & $\begin{array}{l}14 \\
(5.9 \%)\end{array}$ & $\begin{array}{l}4 \\
(1.7 \%)\end{array}$ \\
\hline$P$-Value & 0.542 & & & & 0.016 & & & & 0.516 & & & \\
\hline \multicolumn{13}{|l|}{ Gender } \\
\hline Male & $\begin{array}{l}92 \\
(10.6 \%)\end{array}$ & $\begin{array}{l}550 \\
(63.4 \%)\end{array}$ & $\begin{array}{l}211 \\
(24.3 \%)\end{array}$ & $\begin{array}{l}15 \\
(1.7 \%)\end{array}$ & $\begin{array}{l}257 \\
(29.8 \%)\end{array}$ & $\begin{array}{l}497 \\
(57.6 \%)\end{array}$ & $\begin{array}{l}94 \\
(10.9 \%)\end{array}$ & $\begin{array}{l}15 \\
(1.7 \%)\end{array}$ & $\begin{array}{l}430 \\
(49.8 \%)\end{array}$ & $\begin{array}{l}319 \\
(36.9 \%)\end{array}$ & $\begin{array}{l}82 \\
(9.5 \%)\end{array}$ & $\begin{array}{l}33 \\
(3.8 \%)\end{array}$ \\
\hline Female & $\begin{array}{l}101 \\
(8.8 \%)\end{array}$ & $\begin{array}{l}663 \\
(58.1 \%)\end{array}$ & $\begin{array}{l}349 \\
(30.6 \%)\end{array}$ & $\begin{array}{l}29 \\
(2.9 \%)\end{array}$ & $\begin{array}{l}342 \\
(29.9 \%)\end{array}$ & $\begin{array}{l}686 \\
(60 \%)\end{array}$ & $\begin{array}{l}101 \\
(8.8 \%)\end{array}$ & $\begin{array}{l}14 \\
(1.2 \%)\end{array}$ & $\begin{array}{l}656 \\
(57.5 \%)\end{array}$ & $\begin{array}{l}371 \\
(32.5 \%)\end{array}$ & $\begin{array}{l}79 \\
(6.9 \%)\end{array}$ & $\begin{array}{l}35 \\
(3.1 \%)\end{array}$ \\
\hline$P$-Value & 0.006 & & & & 0.317 & & & & 0.005 & & & \\
\hline \multicolumn{13}{|l|}{ Nationality } \\
\hline Emirati & $\begin{array}{l}55 \\
(13.1 \%)\end{array}$ & $\begin{array}{l}249 \\
(59.4 \%)\end{array}$ & $\begin{array}{l}106 \\
(25.3 \%)\end{array}$ & $\begin{array}{l}9 \\
(2.1 \%)\end{array}$ & $\begin{array}{l}121 \\
(28.9 \%)\end{array}$ & $\begin{array}{l}242 \\
(57.8 \%)\end{array}$ & $\begin{array}{l}51 \\
(12.2 \%)\end{array}$ & $\begin{array}{l}5 \\
(1.2 \%)\end{array}$ & $\begin{array}{l}213 \\
(50.7 \%)\end{array}$ & $\begin{array}{l}152 \\
(36.2 \%)\end{array}$ & $\begin{array}{l}39 \\
(9.3 \%)\end{array}$ & $\begin{array}{l}16 \\
(3.8 \%)\end{array}$ \\
\hline Non Emirati Arab & $\begin{array}{l}96 \\
(7.8 \%)\end{array}$ & $\begin{array}{l}746 \\
(60.4 \%)\end{array}$ & $\begin{array}{l}366 \\
(29.6 \%)\end{array}$ & $\begin{array}{l}28 \\
(2.3 \%)\end{array}$ & $\begin{array}{l}376 \\
(30.5 \%)\end{array}$ & $\begin{array}{l}623 \\
(58.6 \%)\end{array}$ & $\begin{array}{l}114 \\
(9.2 \%)\end{array}$ & $\begin{array}{l}21 \\
(1.7 \%)\end{array}$ & $\begin{array}{l}710 \\
(57.6 \%)\end{array}$ & $\begin{array}{l}388 \\
(31.5 \%)\end{array}$ & $\begin{array}{l}95 \\
(7.7 \%)\end{array}$ & $\begin{array}{l}40 \\
(3.2 \%)\end{array}$ \\
\hline Non Arab & $\begin{array}{l}36 \\
(10.9 \%)\end{array}$ & $\begin{array}{l}206 \\
(62.2 \%)\end{array}$ & $\begin{array}{l}81 \\
(24.5 \%)\end{array}$ & $\begin{array}{l}8 \\
(2.4 \%)\end{array}$ & $\begin{array}{l}92 \\
(28 \%)\end{array}$ & $\begin{array}{l}205 \\
(62.3 \%)\end{array}$ & $29(8.8 \%)$ & $\begin{array}{l}3 \\
(0.9 \%)\end{array}$ & $\begin{array}{l}153 \\
(46.5 \%)\end{array}$ & $\begin{array}{l}142 \\
(43.2 \%)\end{array}$ & $\begin{array}{l}23 \\
(7 \%)\end{array}$ & $\begin{array}{l}11 \\
(3.3 \%)\end{array}$ \\
\hline$P$-Value & 0.025 & & & & 0.431 & & & & 0.003 & & & \\
\hline \multicolumn{13}{|l|}{ City of Residence } \\
\hline Abu Dhabi & $\begin{array}{l}24 \\
(9.1 \%)\end{array}$ & $\begin{array}{l}160 \\
(60.8 \%)\end{array}$ & $\begin{array}{l}68 \\
(25.9 \%)\end{array}$ & $\begin{array}{l}11 \\
(4.2 \%)\end{array}$ & $\begin{array}{l}76 \\
(28.9 \%)\end{array}$ & $\begin{array}{l}148 \\
(56.3 \%)\end{array}$ & $\begin{array}{l}33 \\
(12.5 \%)\end{array}$ & $\begin{array}{l}6 \\
(2.3 \%)\end{array}$ & $\begin{array}{l}135 \\
(51.3 \%)\end{array}$ & $\begin{array}{l}89 \\
(33.8 \%)\end{array}$ & $\begin{array}{l}32 \\
(12.2 \%)\end{array}$ & $\begin{array}{l}7 \\
(2.7 \%)\end{array}$ \\
\hline Dubai & $\begin{array}{l}38 \\
(7.9 \%)\end{array}$ & $\begin{array}{l}312 \\
(64.5 \%)\end{array}$ & $\begin{array}{l}125 \\
(25.8 \%)\end{array}$ & $\begin{array}{l}9 \\
(1.9 \%)\end{array}$ & $\begin{array}{l}135 \\
(28 \%)\end{array}$ & $\begin{array}{l}291 \\
(60.4 \%)\end{array}$ & $\begin{array}{l}53 \\
(11 \%)\end{array}$ & $\begin{array}{l}3 \\
(0.6 \%)\end{array}$ & $\begin{array}{l}249 \\
(51.6 \%)\end{array}$ & $\begin{array}{l}198 \\
(41 \%)\end{array}$ & $\begin{array}{l}24 \\
(5 \%)\end{array}$ & $\begin{array}{l}12 \\
(2.5 \%)\end{array}$ \\
\hline Sharjah & $\begin{array}{l}100 \\
(9.7 \%)\end{array}$ & $\begin{array}{l}609 \\
(59.1 \%)\end{array}$ & $\begin{array}{l}302 \\
(29.3 \%)\end{array}$ & $\begin{array}{l}19 \\
(1.8 \%)\end{array}$ & $\begin{array}{l}326 \\
(31.7 \%)\end{array}$ & $\begin{array}{l}603 \\
(58.6 \%)\end{array}$ & $\begin{array}{l}86 \\
(8.4 \%)\end{array}$ & $\begin{array}{l}14 \\
(1.4 \%)\end{array}$ & $\begin{array}{l}575 \\
(56 \%)\end{array}$ & $\begin{array}{l}332 \\
(32.3 \%)\end{array}$ & $\begin{array}{l}80 \\
(7.8 \%)\end{array}$ & $\begin{array}{l}40 \\
(3.9 \%)\end{array}$ \\
\hline Other Cities & $\begin{array}{l}25 \\
(11.6 \%)\end{array}$ & $\begin{array}{l}123 \\
(56.9 \%)\end{array}$ & $\begin{array}{l}62 \\
(28.7 \%)\end{array}$ & $\begin{array}{l}6 \\
(2.8 \%)\end{array}$ & $\begin{array}{l}58 \\
(26.9 \%)\end{array}$ & $\begin{array}{l}131 \\
(60.6 \%)\end{array}$ & $\begin{array}{l}21 \\
(9.7 \%)\end{array}$ & $\begin{array}{l}6 \\
(2.8 \%)\end{array}$ & $\begin{array}{l}124 \\
(57.7 \%)\end{array}$ & $\begin{array}{l}62 \\
(28.8 \%)\end{array}$ & $\begin{array}{l}21 \\
(9.8 \%)\end{array}$ & $\begin{array}{l}8 \\
(3.7 \%)\end{array}$ \\
\hline$P$-Value & 0.207 & & & & 0.116 & & & & 0.002 & & & \\
\hline \multicolumn{13}{|l|}{ Education } \\
\hline High School or less & $\begin{array}{l}65 \\
(13.7 \%)\end{array}$ & $\begin{array}{l}275 \\
(57.8 \%)\end{array}$ & $\begin{array}{l}126 \\
(26.5 \%)\end{array}$ & $\begin{array}{l}10 \\
(2.1 \%)\end{array}$ & $\begin{array}{l}153 \\
(32.2 \%)\end{array}$ & $\begin{array}{l}264 \\
(55.6 \%)\end{array}$ & $\begin{array}{l}48 \\
(10.1 \%)\end{array}$ & $\begin{array}{l}10 \\
(2.1 \%)\end{array}$ & $\begin{array}{l}247 \\
(52.2 \%)\end{array}$ & $\begin{array}{l}159 \\
(33.6 \%)\end{array}$ & $\begin{array}{l}46 \\
(9.7 \%)\end{array}$ & $\begin{array}{l}21 \\
(4.4 \%)\end{array}$ \\
\hline \multirow[t]{2}{*}{$\begin{array}{l}\text { More than High } \\
\text { school }\end{array}$} & $\begin{array}{l}124 \\
(8.2 \%)\end{array}$ & $\begin{array}{l}931 \\
(61.2 \%)\end{array}$ & $\begin{array}{l}431 \\
(28.3 \%)\end{array}$ & $\begin{array}{l}35 \\
(2.3 \%)\end{array}$ & $\begin{array}{l}440 \\
(29 \%)\end{array}$ & $\begin{array}{l}915 \\
(60.3 \%)\end{array}$ & $\begin{array}{l}144 \\
(9.5 \%)\end{array}$ & $\begin{array}{l}19 \\
(1.3 \%)\end{array}$ & $\begin{array}{l}837 \\
(55.1 \%)\end{array}$ & $\begin{array}{l}522 \\
(34.4 \%)\end{array}$ & $\begin{array}{l}113 \\
(7.4 \%)\end{array}$ & $\begin{array}{l}46 \\
(3 \%)\end{array}$ \\
\hline & & & & & \multicolumn{2}{|c|}{$P$-Value } & \multicolumn{2}{|c|}{0.005} & & & \multicolumn{2}{|c|}{0.206} \\
\hline
\end{tabular}

Table 4 Percentage distribution of the participants who expressed their views about the usefulness of menu labeling $(N=2020)$

\begin{tabular}{lll}
\hline Variables & Percent & Number \\
\hline Very Useful & 47 & 941 \\
Somewhat Useful & 35 & 709 \\
Not Very Useful & 12 & 233 \\
Not At All Useful & 7 & 136 \\
\hline
\end{tabular}

Table 5 Percentage distribution of the participants who expressed their views about the usefulness of menu labeling $(N=2020)$

\begin{tabular}{lll}
\hline Variables & Percent & Number \\
\hline Favor & 76 & 1539 \\
Oppose & 7 & 131 \\
Neither & 17 & 343 \\
\hline
\end{tabular}


Table 6 Percentage distribution of the demographic variables of the participants as associated with attitude towards menu labeling (2020)

\begin{tabular}{|c|c|c|c|}
\hline Variable & $\begin{array}{l}\text { Favor } \\
\%\end{array}$ & $\begin{array}{l}\text { Oppose } \\
\%\end{array}$ & $\begin{array}{l}\text { Neither } \\
\%\end{array}$ \\
\hline \multicolumn{4}{|l|}{ Age, Y } \\
\hline $18-24$ & 72.2 & 7.5 & 20.3 \\
\hline $25-39$ & 81.9 & 5.6 & 12.5 \\
\hline $40+$ & 85 & 3.3 & $(11.7$ \\
\hline$P$-Value & 0.000 & & \\
\hline \multicolumn{4}{|l|}{ Gender } \\
\hline Male & 73.1 & 7.3 & 19.7 \\
\hline Female & 79 & 5.9 & 15 \\
\hline$P$-Value & 0.007 & & \\
\hline \multicolumn{4}{|l|}{ Nationality } \\
\hline Emirati & 76.8 & 8.8 & 14.5 \\
\hline Non Emirati Arab & 76.9 & 5.8 & 17.3 \\
\hline Non Arab & 74.3 & 5.4 & 20.2 \\
\hline$P$-Value & 0.071 & & \\
\hline \multicolumn{4}{|l|}{ City of Residence } \\
\hline Abu Dhabi & 79.8 & 5.7 & 14.4 \\
\hline Dubai & 74.8 & 5.8 & 19.3 \\
\hline Sharjah & 75.7 & 7.1 & 17.2 \\
\hline Other Cities & 80.9 & 5.1 & 14 \\
\hline$P$-Value & 0.353 & & \\
\hline \multicolumn{4}{|l|}{ Education } \\
\hline High school or less & 74.7 & 6.8 & 18.6 \\
\hline More than High school & 77.2 & 6.3 & 16.5 \\
\hline$P$-Value & 0.520 & & \\
\hline
\end{tabular}

labeled restaurant, with the majority of the young participants responded positively to menu-labeling. Moreover, it was found that participants who had more than high school education answered the daily caloric requirement questions correctly. Krukowski et al. [15] has reported that college students want nutrition labels and would use them to inform their food purchasing decisions.

In our study, the majority of the participants, and women in particular, had a favorable response to menu labeling and correctly estimated the caloric requirements for active women and men. The majority of the

Table 7 Percentage distribution of the participants who are likely to eat at a labeled restaurant $(N=2020)$

\begin{tabular}{lll}
\hline & Percent & Number \\
\hline Variable & & \\
More Likely & 48 & 961 \\
Less Likely & 24 & 486 \\
Neither & 28 & 570 \\
\hline
\end{tabular}

Table 8 Percentage distribution of the demographic variables of the participants as associated with their likeliness towards dining in a menu labeled restaurant (2020)

\begin{tabular}{|c|c|c|c|}
\hline & More Likely & Less Likely & Neither \\
\hline \multicolumn{4}{|l|}{ Age, Y } \\
\hline $18-24$ & $507(42.5 \%)$ & $315(26.4 \%)$ & $370(31.0 \%)$ \\
\hline $25-39$ & $308(54.0 \%)$ & $120(21.1 \%)$ & $142(24.9 \%)$ \\
\hline $40+$ & $137(57.1 \%)$ & $47(19.6 \%)$ & $56(23.3 \%)$ \\
\hline$P$-Value & 0.000 & & \\
\hline \multicolumn{4}{|l|}{ Gender } \\
\hline Male & $395(45.5 \%)$ & $213(24.5 \%)$ & $260(30.0 \%)$ \\
\hline Female & $564(49.3 \%)$ & $271(23.7 \%)$ & $310(27.1 \%)$ \\
\hline$P$-Value & 0.217 & & \\
\hline \multicolumn{4}{|l|}{ Nationality } \\
\hline Emirati & $205(48.6 \%)$ & $100(23.7 \%)$ & $117(27.7 \%)$ \\
\hline Non Emirati Arab & $596(48.2 \%)$ & $304(24.6 \%)$ & $336(27.2 \%)$ \\
\hline Non Arab & $145(43.8 \%)$ & $72(21.8 \%)$ & $114(34.4 \%)$ \\
\hline$P$-Value & 0.135 & & \\
\hline \multicolumn{4}{|l|}{ City of Residence } \\
\hline Abu Dhabi & $112(42.7 \%)$ & $84(32.1 \%)$ & $66(25.2 \%)$ \\
\hline Dubai & $238(49.1 \%)$ & $95(19.6 \%)$ & $152(31.3 \%)$ \\
\hline Sharjah & $496(48.0 \%)$ & $241(23.3 \%)$ & $297(28.7 \%)$ \\
\hline Other Cities & $107(49.8 \%)$ & $52(26.5 \%)$ & $51(23.7 \%)$ \\
\hline$P$-Value & 0.007 & & \\
\hline \multicolumn{4}{|l|}{ Education } \\
\hline High school or less & $205(43.0 \%)$ & $116(24.3 \%)$ & $156(26.7 \%)$ \\
\hline More than High school & $750(49.3 \%)$ & $365(24.0 \%)$ & $407(28.2 \%)$ \\
\hline$P$-Value & 0.022 & & \\
\hline
\end{tabular}

respondents in this study expressed their intention to choose to eat at a menu-labeled restaurant. Similarly, Din [16] showed that that women were more interested in menu labeling, and believed that it aid in regulating their intake than men.

It is worth noting that the majority of the respondents in this study eat more frequently at restaurants. Mussaiger [17] had reported that the proportion of obesity increased to reach $52.7 \%$ among those who eat outside the home for more than 5 times per week since the foods are eaten outside the home is more likely to be high in total energy, total fat, saturated fat.

Several prospective studies have reported that eating more frequently away from home in restaurants, is associated with weight gain over time compared to infrequent restaurant eating $[18,19]$. Therefore, a better understanding of public perceptions about calorie posting may encourage policy makers to adopt this low-cost policy tool in order to educate consumers to make healthier food choices.

It has been reported that people who used menu labeling to determine calorie content consumed significantly 
fewer calories during a meal compared with people who did not use menu labeling [20]. Roberto et al. [7] studied the impact of menu labeling on food choices and intake and found that research participants who received caloric-labeled menus consumed $14 \%$ fewer calories than those who received menus without calorie labeling. An average reduction of $100 \mathrm{cal}$ per meal resulted among restaurant patrons who ordered reduced-calorie meals in response to calorie postings on the menus [21].

To our knowledge, the current study is the first to assess consumers' understanding of overall daily energy requirements and the perceived effectiveness of calorie posting in dine-in restaurants in the UAE. The results from this study may form the basis of future strategies in mandating calorie posting in dine-in restaurants as well as fast food chains in the United Arab Emirates.

Furthermore, menu-labeling may serve to encourage restaurants to highlight lower calorie options and/or introduce healthier options. Given the positive findings of intention to choose restaurants with labeled menus, some restaurants may also begin providing caloric information voluntarily.

This study has some limitations including, firstly, that as a cross-sectional study, it only allows associations to be addressed. Second, as Bleich \& Pollack [4] stated, the range of calories in the correct response category for the caloric knowledge question was broad; thus, the finding of relatively high caloric knowledge may be biased upwards. Third, given that the correct answer to the caloric knowledge questions was the same for all groups (e.g. moderately active men, moderately active women, and inactive adults), some participants may have assumed that the answer must change across groups. This would bias our results downwards and may partially explain our finding of low caloric literacy about inactive adults.

\section{Conclusion}

The results of this study encourage further research concerning the implementation of menu labeling in restaurants. This is especially significant given that this is a new concept in the UAE. Additionally, research studies of nutrient and calorie awareness are needed to ensure that UAE residents understand calorie requirements and how to read and apply nutrition facts on product labels to their consumption practices. It would also be of interest to conduct an experimental study comparing two restaurants - one with menu labeling and one without by measuring the amount of calories purchased by individuals. Studies should identify how and where menu labeling can be best presented to most effectively help consumers make healthier choices and lower their caloric intake.

Given that there is an increasing frequency of restaurant visitors in UAE, mandating calorie posting in dine- in, as well as fast food restaurants may be a useful policy tool for promoting appropriate energy intake, to help consumers make food choices in restaurants, contributing to lower rates of obesity.

\section{Acknowledgements}

We wish to thank Nama Al-Mosa, Sumaya Hashem, Jawaher Hilal, Ruba ElHourani, and Deena Khales for their assistance in data collection.

Funding

There is no source of funding. The authors self-funded the research.

Availability of data and materials

The datasets during and/or analysed during the current study will be available from the corresponding author on reasonable request.

\section{Authors' contribution}

$\mathrm{HR}$ and $\mathrm{EF}$ and RO contributed to conception and design, acquisition of data, and interpretation of data. EF was involved in analyzing data and drafting manuscript. HR and RO have been involved in revising it critically for important intellectual content. HR gave final approval of the version to be published. Each author have participated sufficiently in the work to take public responsibility for appropriate portions of the content; and agree to be accountable for all aspects of the work in ensuring that questions related to the accuracy or integrity of any part of the work are appropriately investigated and resolved. All authors read and approved the final manuscript.

\section{Competing interests}

The authors declare that they have no competing interests.

\section{Consent for publication}

Not applicable.

Ethics approval and consent to participate

The study was conducted in the cities of Sharjah and Dubai in the United Arab Emirates and approved by Ethical Committee at the University of Sharjah. Consent was obtained from participants before they were interviewed.

\section{Author details}

'Department of Clinical Nutrition and Dietetics, College of Health Sciences/ Sharjah Institute for Medical Research, University of Sharjah, P. O Box 27272, Sharjah, United Arab Emirates. ${ }^{2}$ Dubai Health Authority, Dubai, United Arab Emirates.

Received: 23 November 2016 Accepted: 10 January 2017

Published online: 27 February 2017

\section{References}

1. Al-Nohair S. Obesity in gulf countries. IJHSR (Qassim). 2014;8(1):79-83.

2. Health Authority Abu Dhabi. Health statistics: reliable excellence in healthcare. 2014. www.haad.ae/statistics. Accessed June 2016.

3. Simon P, Jarosz CJ, Kuo T, Fielding JE. Menu labeling as a potential strategy for combating the obesity epidemic: a health impact assessment, published by division of chronic disease and injury prevention. Los Angeles County: Department of Public Health; 2008. p. 1-8.

4. Bleich SN, Pollack K. The publics' understanding of daily caloric recommendations and their perceptions of calorie posting in chain restaurants. BMC Public Health. 2010;10(121):1471-2458.

5. Bleich SN, Wolfson JA, Jarlenski MP, Block JP. Restaurants with calories displayed on menus Had lower calorie counts compared to restaurants without such labels. Health Aff. 2015;34(11):1877-84.

6. Lee-Kwan SH, Pan L, Maynard LM, McGuire LC, Park S. Factors associated with self-reported menu-labeling usage among US adults. J Acad Nutr Diet. 2016;15:1813-4.

7. Roberto CA, Larsen PD, Agnew H, Baik J, Brownell KD. Evaluating the impact of menu labeling on food choices and intake. Am J Public Health. 2010;100:312-8. 
8. Spicer D, Brissette I, Lowenfels A, Noble C. Predictors of total calories purchased at fast-food restaurants: restaurant characteristics, calorie awareness, and use of calorie information. J Nutr Educ Behav. 2013;45:404-11.

9. Huang C, Dumanovsky T. A brief overview of New York City's calorie labeling regulations and evaluation. Nutr Today. 2010;45:226-8.

10. Burton S, Creyer E, Kees J, Huggins K. Attacking the obesity epidemic: the potential health benefits of providing nutrition information in restaurants. Am J Public Health. 2006;96:1669-75.

11. Lin B, Guthrie J. Nutritional quality of food prepared at home and away from home, 1977-2008. U.S. Department of Agriculture, Economic Research Service; 2012.

12. Block JP, Condon S, Mullen J, Linakis S, Rifas'Shiman S, Gillman M. Consumers' estimation of calorie content at fast food restaurants: cross sectional observational study. Br Med J. 2014;10:f2907.

13. UAE Ministry of Education. Higher education in UAE. Washington: Publications of Embassy of United Arab Emirates; 2010. p. 1-3. www.uaeembassy.org.

14. Department of Health and Human Services and U.S. Department of Agriculture. Dietary guidelines for Americans. Washington DC: HHS and USDA; 2010.

15. Krukowski R, Harvey-Berino J, Kolodinsky J, Narsana RT, Desisto TP. Consumers may not use or understand calorie labeling in restaurants. J Acad Nutr Diet. 2006;106(6):917-20.

16. Din N, Zahari MSM, Shariff SM, Universiti Teknologi M. Nutritional labelling in Malaysian full service restaurant menu. J Asian behav Studies. 2011;1(3):51-9.

17. Musaiger AO. Overweight and obesity in eastern Mediterranean region: prevalence and possible causes. J Obes. 2011;407237.

18. Bruemmer B, Krieger J, Saelens BE, Chan N. Energy, saturated fat, and sodium were lower in entrées at chain restaurants at 18 months compared with 6 months following the implementation of mandatory menu labeling regulation in King County, Washington. J Acad Nutr Diet. 2012;112(8):1169-76.

19. Thompson O, Ballew C, Resnicow K, Must A, Bandini L, Cyr H, Dietz W. Food purchased away from home as a predictor of change in BMl z-score among girls. Int J Obes. 2004;28(2):282-9.

20. Duffey K, Gordon-Larsen P, Jacobs D, Williams O, Popkin B. Differential associations of fast food and restaurant food consumption with $3-y$ changes in body mass index: the coronary artery risk development in young adults study. Am J Clin Nutr. 2007:85:201-8.

21. Kuo T, Jarosz CJ, Simon P. Fielding JE. Menu labeling as a potential strategy for combating the obesity epidemic: a health impact assessment. Am J Public Health. 2009;99(9):1680-6.

\section{Submit your next manuscript to BioMed Central and we will help you at every step:}

- We accept pre-submission inquiries

- Our selector tool helps you to find the most relevant journal

- We provide round the clock customer support

- Convenient online submission

- Thorough peer review

- Inclusion in PubMed and all major indexing services

- Maximum visibility for your research

Submit your manuscript at www.biomedcentral.com/submit

C Biomed Central 